\title{
The Function of Auxiliary Safety Management Subsystem of Railway Permanent Way Used in the State Analysis of the Track
}

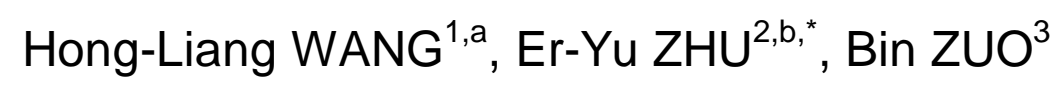

${ }^{1,2,3}$ School of civil engineering, Beijing Jiaotong University, Beijing 100044, China

whl.981@163.com, beyzhu@bjtu.edu.cn

${ }^{*}$ Corresponding author

Keywords: Auxiliary Management Subsystem, State Analysis of the Track, Management of Railway, Permanent Way.

\begin{abstract}
In this paper, the function of auxiliary safety management subsystem of railway permanent way which used in the state analysis of the track is introduced. The content and process of the auxiliary management subsystem used in the state analysis of the track; the relationship between all components of the system is also shown clearly by the function relationship diagram.
\end{abstract}

\section{Introduction}

The importance of the role that the railway played in the national economy is un-doubt, so it is always a strategic task to ensure the normal operation of the railways. Railway permanent way is a very tedious and complicated work since the birth of the railway, so there isn't a scientific management method in the early days when the railway was born. The development of the railway permanent way management system based on computer platform has become possible with the improving of the computer technology [1-5].

The function of auxiliary safety management subsystem of railway permanent way used in the state analysis of the track is introduced in this paper.

\section{The Introduction of the State Analysis of the Railway Track}

There are two works including in the state analysis of the railway track: to assess the testing data; to show the results of the comparison between the current data and the existing data according to the current management standards.

There are three functions when the auxiliary safety management subsystem of railway permanent way used in the state analysis of the track.

(1) The inspection report of the state test;

(2) The analysis of the state test;

(3) Comprehensive analysis of the state test.

\section{The Inspection Report of the State Test}

The function of the inspection report of the state test is used in the collection of the transfinite data, its function can help the user to control the situation of the disease which can provide the help for the repairing. The state of the track and kinds of test analysis also can be gotten in this report which provided by the function of the auxiliary management subsystem.

This function is the basis for the repair plan which can also provide the transfinite data for the implementation of other function module. The reports which can be provided in the inspection report of the state test are shown in Fig.1 


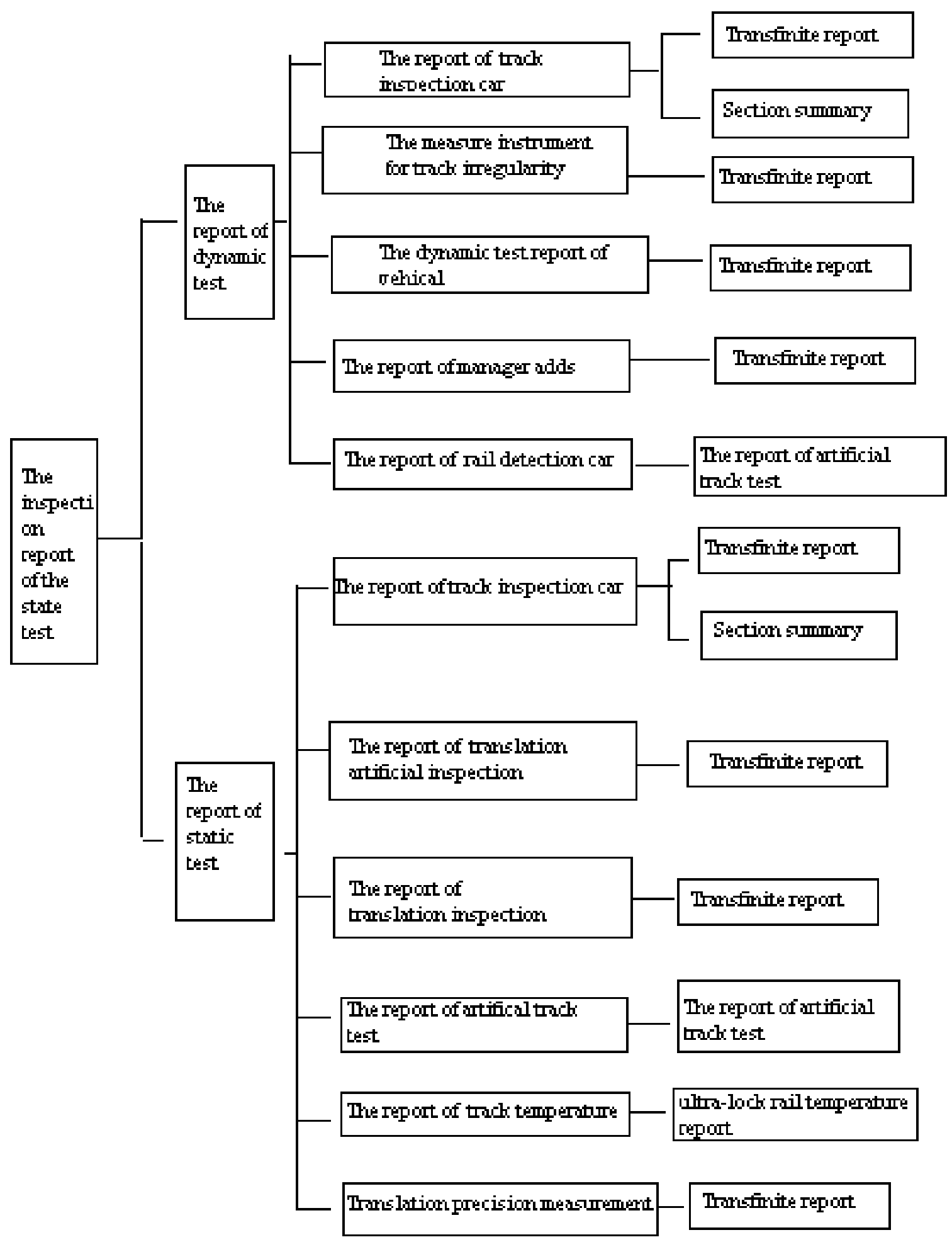

Fig. 1 The Detection Report of the Track State

\section{The Analysis of the State Test}

The function is used in the railway state comprehensive evaluation and the investigation of the rule which exist in changing state of the track by analyzing all the evaluation data. And this work will help to implement other function module. This function of this part is shown in Fig.2. 


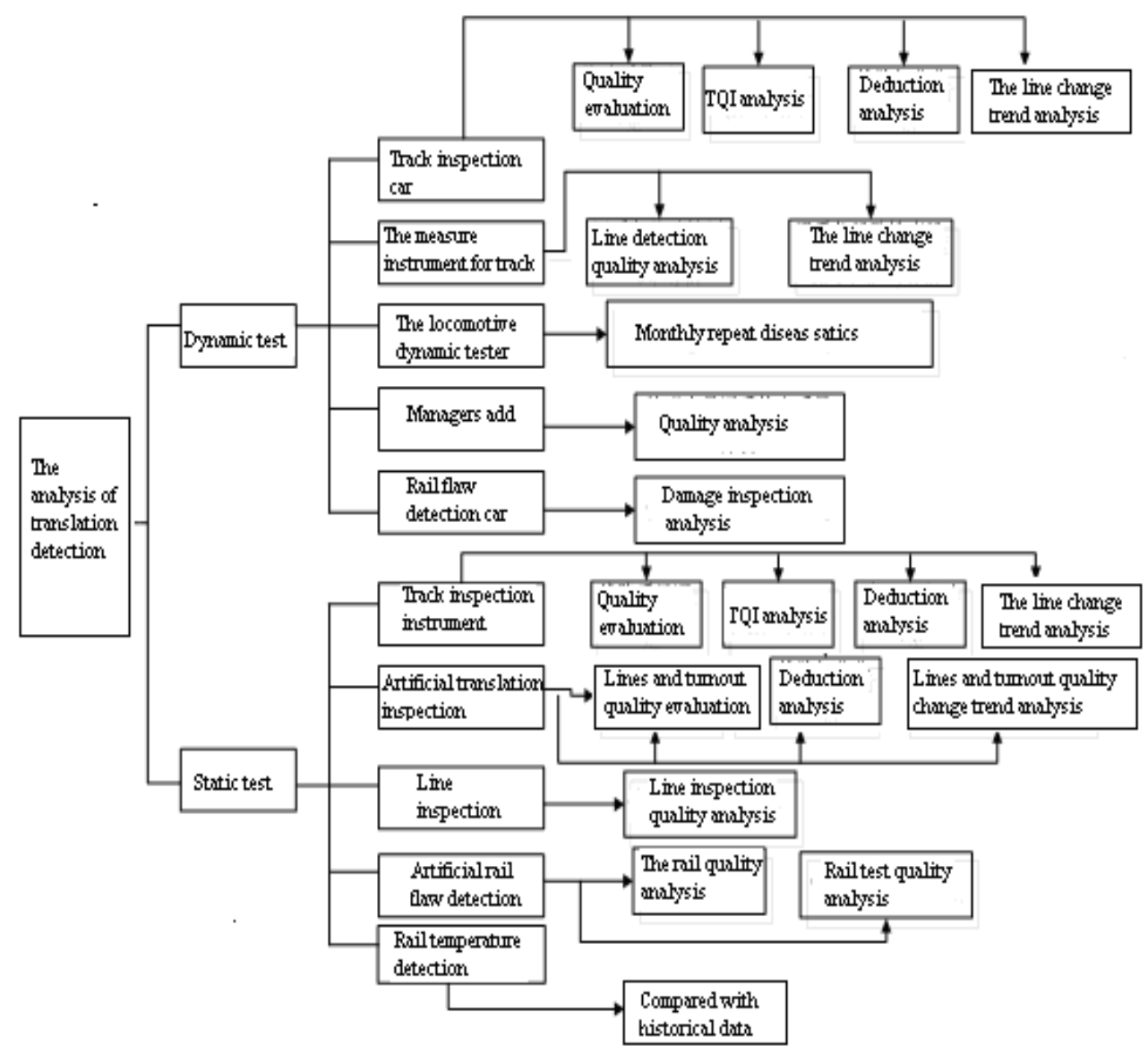

Fig. 2 Line Detection Data Analysis

\section{Static Test Data Analysis}

There are many functions can be implemented through the data which is collected by the track inspection instrument. For example: the translation quality assessment, the translation quality index (TQI) comparative analysis, the deduction scheme analysis, the distribution of the deduction scheme, the playback of the track instrument data, the TQI historical data comparison and the changing trend analysis of the translation.

The functions which can be implemented by the artificial translation inspection data are these, such as: the quality assessment of the translation and turnouts, the analysis of the deduction scheme in the artificial translation and turnout inspection, the changing trend analysis of the turnout quality.

The translation inspection data are mainly used in the analysis of the translation inspection quality.

The quality analysis of the track and the detection work can be implemented by the artificial rail failure detection data.

\section{Dynamic test Data Analysis}

The dynamic test data analysis has the similar function with the static test data analysis, so the functions are not given out here.

According to dynamic and static test data, a comprehensive analysis of the priority diseases can be achieved, and then the quality evaluation system after repaired can be established in order to strengthen the management of the quality for the repaired work [6].

\section{The Comprehensive Analysis of the Translation State Detection}

The comprehensive analysis of the translation state detection mainly reflects the comparison of the maintenance data which are located in the front and back $50 \mathrm{~m}$ of the disease position. The analysis of the repairing quality is carried on by comparing the test data within maintenance, and it will help the manager 
to master the actual state of the translation. The relationship between the analysis of translation detection and other module functions is shown in Fig. 3.

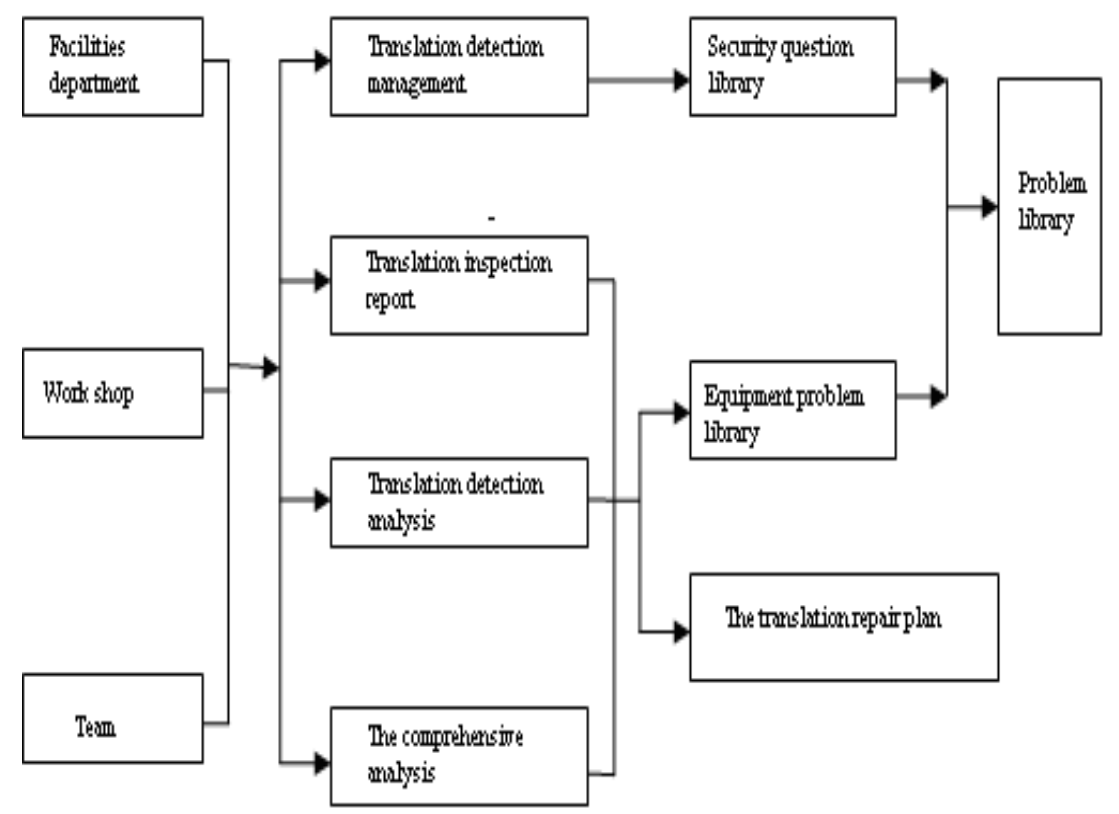

Fig. 3 Association Graph with Translation State Analysis and Each Module Functions

\section{Summary}

The function of auxiliary safety management subsystem of railway permanent way used in the state analysis of the track is introduced in the paper. A correlation graph is given out, which makes the function of the system used in the analysis of the translation state more clearly. This work will make the system more clear for managers, and will greatly improve the efficiency in studying this kind of technology.

\section{Acknowledgement}

The authors wish to acknowledge the support and motivation provided by Science and Technology Research and Development Plan of Railway Ministry (No.2012G002-5), Specialized Research Fund for the Doctoral Program of Higher Education of China(No.20110009110016),Science and Technology Research and Development Plan of China Railway Corporation (No.Z2013-G004).

\section{References}

[1]Jun Liu. Railway works testing data integrated information platform for research and implementation [D]Beijing: Beijing Jiaotong university, 2006.

[2]Xia Wu. Railway track state analysis method research [D]. Beijing: Beijing Jiaotong University, 2008.

[3]Jibing Lou. Railway track parameters test and data processing technology research [D]. Changsha: Central south university, 2007

[4]Xinyu Xun. Railway works safety production management aided decision system [D]. Beijing: Beijing Jiaotong University, 2006.

[5]Changqing Yuan,Yugang huang, Hongkai Niu. Build a railway line equipment inspection unified database [J]. Journal of traffic engineering and national defense technology, 2005, (4): 42-44.

[6]Yuchun Huang. Urban rail transit works management methods and technology research [D]. Shanghai: Tongji University, 2007. 